\title{
Perfil farmacoterapêutico de pacientes renais crônicos hemodialíticos em uma clínica da Bahia
}

\author{
Pharmacotherapeutic profile of chronic hemodialytic kidney patients \\ in a Bahia clinic
}

Perfil farmacoterapéutico de pacientes renales crónicos hemodialíticos en una clínica Bahia

Lucas Brasileiro LEMOS ${ }^{(1)}$

Gabriela Silva MORAES ${ }^{(2)}$

Gisele da Silveira $\operatorname{LEMOS}^{(2)}$

Adriana Alves NERY(3)

Recebido: 24 abr 2020

Revisado: 02 jun 2020

Aceito: 01 jul 2020

Autor de correspondência:

Gisele da Silveira Lemos

giselesilveirasemos@gmail.com

Conflito de interesses:

Os autores declaram não haver nenhum interesse profissional ou pessoal que possa gerar conflito de interesses em relação a este manuscrito.

\author{
(1)Universidade Estadual do Sudoeste da Bahia, Curso de Medicina, Departamento de Saúde II, \\ Jequié, BA, Brasil. \\ (2)Universidade Estadual do Sudoeste da Bahia, Curso de Farmácia, Departamento de Ciências e \\ Tecnologias, Jequié, BA, Brasil. \\ (3)Universidade Estadual do Sudoeste da Bahia, Curso de Enfermagem, Departamento de Saúde II, \\ Jequié, BA, Brasil.
}

\section{Resumo}

A doença renal crônica (DRC) configura-se como um problema de saúde pública devido a prevalência e morbimortalidade crescentes. As múltiplas comorbidades associadas a DRC exigem um manejo adequado dos medicamentos mitigando riscos e problemas farmacoterapêuticos. Assim, esse estudo teve como objetivo descrever o perfil farmacoterapêutico e clínico de pacientes renais crônicos hemodialíticos e caracterizar seu modo de utilização de medicamentos: prescritos e por automedicação. Trata-se de um estudo transversal realizado em um centro de hemodiálise no Estado da Bahia, através da aplicação de um formulário, sendo os pacientes selecionados em março de 2015. Dentre os participantes, predominaram os do sexo masculino $(64,1 \%)$, com média de idade $50,5 \pm 14,9$ anos. Entre os participantes $83,5 \%$ declararam renda familiar mensal de até 2 salários mínimos, 20\% nunca estudaram, 48,2\% estavam em diálise por períodos compreendidos entre 1 e 5 anos e 92,9\% não possuíam plano de saúde. Hipertensão Arterial Sistêmica foi a causa mais frequente da perda da função renal (57,8\%). O número

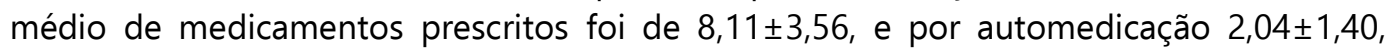
sendo que $39,4 \%$ dos participantes declararam ter se automedicado em algum momento da vida. O medicamento mais frequentemente prescrito foi a eritropoetina $(10,6 \%)$ e o mais frequente dentre os classificados como de automedicação o calcitriol (9,6\%), influenciado por falha na comunicação entre o prescritor e paciente. Os dados apresentados servem de subsídio para um aprimoramento da assistência ao paciente com DRC, principalmente em relação ao uso racional de medicamentos.

Descritores: Diálise Renal; Farmacoepidemiologia; Uso de Medicamentos. 


\title{
Abstract
}

Chronic kidney disease (CKD) is a public health problem due to the increasing prevalence and morbidity and mortality. The multiple comorbidities associated with CKD require proper drug management to mitigate risks and pharmacotherapeutic problems. Thus, this study aimed To describe the pharmacotherapeutic and clinical profile of hemodialysis chronic renal patients and to characterize their use of medications: prescribed and by self-medication. This is a cross-sectional study conducted in a hemodialysis center in the state of Bahia, through the application of a form, and the patients were selected in March 2015. Among the participants, predominated males (64.1\%), with a mean age of $50.5 \pm 14.9$ years. Among the participants $83.5 \%$ reported monthly family income of up to 2 minimum wages, $20 \%$ never studied, $48.2 \%$ were on dialysis for periods between 1 and 5 years and $92.9 \%$ had no health insurance. Systemic Arterial Hypertension was the most frequent cause of loss of renal function (57.8\%). The average number of prescription drugs was $8.11 \pm 3.56$, and by selfmedication $2.04 \pm 1.40$, and $39.4 \%$ of participants reported self-medication at some point in their lives. The most frequently prescribed drug was erythropoietin (10.6\%) and the most frequent among the self-medication classified as calcitriol (9.6\%), influenced by a lack of communication between the prescriber and the patient. The data presented serve as subsidy for improvement of care for patients with CKD, particularly in relation to the rational use of medicines.

Keywords: Renal Dialysis; Pharmacoepidemiology; Drug Utilization.

\section{Resumen}

La enfermedad renal crónica (ERC) es un problema de salud pública debido a su creciente prevalencia, morbilidad y mortalidad. Las múltiples comorbilidades asociadas con la ERC requieren un manejo adecuado de la medicación, mitigando los riesgos y problemas farmacoterapéuticos. Por lo tanto, este estudio tuvo como objetivo describir el perfil farmacoterapéutico y clínico de los pacientes con hemodiálisis crónica y caracterizar su uso de medicamentos: prescritos y automedicados.Este es un estudio transversal realizado en un centro de hemodiálisis en el estado de Bahía, mediante la aplicación de un formulario, con pacientes seleccionados en marzo de 2015. Entre los participantes, predominantemente hombres $(64,1 \%)$, con edad media $50.5 \pm 14.9$ años. Entre los participantes, el 83.5\% declaró un ingreso familiar mensual de hasta 2 salarios mínimos, el $20 \%$ nunca estudió, el 48.2\% estaba en diálisis por períodos entre 1 y 5 años y el $92.9 \%$ no tenía seguro médico. La hipertensión arterial sistémica fue la causa más frecuente de pérdida de la función renal (57.8\%). El número promedio de medicamentos recetados fue $8.11 \pm 3.56$, y para la automedicación $2.04 \pm 1.40$, con el 39.4\% de los participantes declarando que se habían automedicado en algún momento de sus vidas. La medicación prescrita con mayor frecuencia fue la eritropoyetina $(10,6 \%)$ y la más frecuente entre las clasificadas como automedicación fue el calcitriol $(9,6 \%)$, influenciado por la falta de comunicación entre el prescriptor y el paciente. Los datos presentados sirven como base para mejorar la asistencia a pacientes con ERC, especialmente en relación con el uso racional de medicamentos.
\end{abstract}

Palabras-claves: Diálisis Renal; Farmacoepidemiología; Utilización de Medicamentos.

\section{Introdução}

No último censo de diálise realizado pela Sociedade Brasileira de Nefrologia - SBN, em 2017, estimou-se que existem cerca de 126.583 pacientes em terapia dialítica, sendo que destes, cerca de 93,1\% encontram-se em hemodiálise. Somente no estado da Bahia, a taxa de prevalência de insuficiência renal crônica em diálise foi de 518 pacientes por milhão de população neste mesmo ano. ${ }^{1}$

Relata-se um aumento na prevalência destes pacientes, também nos Estados Unidos e em outros países desenvolvidos da Europa e Ásia, ${ }^{1}$ mas a mortalidade continua alta, apesar dos avanços tecnológicos obtidos no tratamento hemodialítico. ${ }^{2}$ Isto pode ser explicado, em 
parte, pelo fato da idade e número de comorbidades serem cada vez maiores nesta população.

O diagnóstico e manejo da doença renal crônica (DRC) e de suas complicações fazem da polifarmácia, um fenômeno de alta prevalência e uma consequência natural da prestação do cuidado aos pacientes renais crônicos dialíticos. ${ }^{3}$ Com este número elevado de medicamentos utilizados por estes pacientes ${ }^{4-6}$ aumenta-se 0 risco de problemas farmacoterapêuticos, com implicações de custo no sistema de saúde. ${ }^{7}$ Portanto, os estudos farmacoepidemiológicos em renais crônicos são de grande importância, uma vez que servirão de subsídio para orientar um melhor planejamento por parte dos gestores, com foco em prevenção na atenção primária a saúde e também para a assistência multiprofissional a ser ofertada a esta população. ${ }^{1,8}$

Estudos farmacoepidemiológicos, devido à ampla extensão territorial brasileira e aos diferentes perfis farmacoterapêuticos regionais, se tornam cada vez mais necessários e são ferramentas essenciais para a adoção de medidas estratégicas que garantem o acesso e o uso racional desse importante recurso terapêutico nas diferentes esferas da assistência à saúde. ${ }^{9}$ A qualidade de serviços de saúde pode ser melhor planejada e obter melhores resultados também por meio da análise dos dados de tais estudos, e o desenvolvimento de práticas de cuidado de saúde voltadas a uma melhor segurança dos pacientes se tornará mais evidente. ${ }^{8}$

$\mathrm{Na}$ última década tem ocorrido um crescimento proeminente dos estudos farmacoepidemiológicos, principalmente em decorrência de algumas limitações dos ensaios clínicos randomizados como, por exemplo, a omissão ou sub-representação de populações chaves como a dos pacientes renais crônicos. ${ }^{10}$ Neste contexto, os objetivos deste estudo foram descrever o perfil farmacoterapêutico e clínico de pacientes renais crônicos hemodialíticos e caracterizar seu modo de utilização de medicamentos: prescritos e por automedicação.

\section{Metodologia}

\section{Tipo de estudo e local da pesquisa}

Foi realizado um estudo transversal, em uma clínica privada, conveniada com o Sistema Único de Saúde - SUS para atendimento a pacientes renais crônicos, localizada no estado da Bahia, que prestava assistência em diálise, para cerca de 250 pacientes no período de março de 2015. A população atendida por essa clínica é regional, abrangendo 27 municípios pertencentes ao Núcleo de Saúde Sul da Bahia.

\section{Amostra e critérios de inclusão e exclusão}


ISSN 2179-6750

Do total de pacientes, compuseram a amostra do estudo, 189 indivíduos, por atenderem aos seguintes critérios de inclusão: pacientes hemodialíticos crônicos, com tempo de tratamento superior a 1 ano e idade maior ou igual a 18 anos. Destes préselecionados, apenas 170 responderam ao formulário aplicado. Dos 19 não respondedores, 5 se recusaram a participar, 6 não conseguiram responder ao instrumento, e dos outros que não participaram, 2 foram por óbito, 2 por transferência para outro serviço de diálise, 2 por abandono de tratamento, 1 faltou nos dias agendados e 1 migrou para diálise peritoneal no período.

\section{Instrumentos e coleta de dados}

Os participantes do estudo, após a assinatura do Termo de Consentimento Livre e Esclarecido, responderam a um formulário preenchido por profissionais previamente treinados (uma enfermeira e uma farmacêutica), não pertencentes à equipe de trabalho do centro de diálise.

Após o treinamento, foi realizado um estudo piloto para adequação do instrumento de coleta de dados e calibração dos entrevistadores, por meio da aplicação do formulário junto aos pacientes do mesmo centro de tratamento, que foram inclusos na população selecionada, pelo fato de não terem sido necessárias mudanças na estrutura do formulário. Este foi desenvolvido pelos pesquisadores com a ajuda de uma equipe multidisciplinar tomando, como referência, dados obtidos após uma leitura maciça do tema em todo referencial bibliográfico, utilizando principalmente modelos de questionários prévios como os do Estudo SABE ${ }^{11}$ e PNAUM ${ }^{12}$, além do elaborado por Baldoni, ${ }^{13}$ em sua dissertação de mestrado.

\section{Variáveis}

O formulário foi composto por perguntas referentes a dados sociodemográficos (sexo, idade, raça, escolaridade, renda familiar mensal, religião, estado civil, ocupação, com quem vive), além de outras questões específicas relacionadas a sua saúde e aos serviços de saúde como a data da última consulta médica (considerada esta, apenas quando o paciente tenha recebido atendimento em consultório médico), a presença de comorbidades como diabetes mellitus (DM) e hipertensão arterial sistêmica (HAS) concomitantes à doença renal, assim como hábitos de vida (independente do tratamento hemodialítico) e dados sobre o uso de medicamentos. Quando os participantes não souberam ou não conseguiram responder o nome dos medicamentos de que faziam uso, a pergunta foi dirigida aos cuidadores. 
Quanto aos medicamentos consumidos, foram aceitos, se apresentadas as bulas ou embalagens ou prescrições dos mesmos, semelhante ao estudo realizado em Viçosa, MG, ${ }^{14}$ e quando o paciente relatava o uso de algum medicamento prescrito e não tinha apresentado bula, embalagem ou prescrição, esse era considerado, se estivesse presente na prescrição eletrônica, no prontuário eletrônico do paciente.

Foram considerados medicamentos prescritos, aqueles usados em qualquer situação, nos últimos 30 dias, apresentados em receituários médicos, ou quando estes não eram apresentados, e o paciente relatava o uso ou trazia bulas ou embalagens, mas tais medicamentos eram encontrados prescritos no prontuário eletrônico do participante, em uma posterior conferência realizada pelo pesquisador. Quando os medicamentos não se enquadraram nestas situações, foram classificados como automedicação, ou não prescritos, considerando o período recordatório de 30 dias, mesmo que fosse por continuidade de um fármaco previamente prescrito, mas que, por alguma falha de comunicação entre os prescritores e os pacientes, não tivesse sido interrompido o uso.

Optou-se por não contabilizar a medicação heparina no número de medicamentos usados, pois é utilizada por todos os pacientes durante suas sessões de hemodiálise, para evitar coagulação do filtro, exceto se contraindicação momentânea.

As medicações foram classificadas como "Prescritas ou Não-prescritas" e "de uso na clínica, ou no domicílio". Apesar de o cloridrato de sevelamer ser usado em domicílio, foi enquadrado apenas como de uso na clínica, pelo fato de sua prescrição ocorrer somente junto com os outros considerados como de alto custo e de uso especial (usados na clínica). Dúvidas e itens do formulário não preenchidos na primeira entrevista foram complementados em um segundo momento. Ambas foram previamente agendadas, e os pacientes e/ou acompanhantes recebiam durante a sessão de diálise que antecedia a do dia da entrevista, um lembrete impresso, onde as instruções eram cuidadosamente explicadas.

Os medicamentos em uso e seus respectivos princípios ativos foram classificados em cada especialidade, de acordo com a Classificação Terapêutica Anatômica - ATC, da Organização Mundial de Saúde, ${ }^{15}$ sendo que medicamentos usados como terapia complementar, homeopática ou fitoterápica, em geral, não estão incluídos nesta classificação.

\section{Análise dos dados}

Para tabulação dos dados foi utilizado o programa Epidata versão 3.1 (Epidata Association, Odense, Dinamarca). O processamento e a análise dos dados foram realizados utilizando o programa SPSS (Statistical Package for the Social Sciences) versão 21.0. A 
estatística descritiva foi apresentada por meio de frequências (absoluta e relativa), médias e desvio-padrão.

\section{Considerações éticas}

Em todos os aspectos seguidos, primou-se por respeitar as questões éticas que envolvem pesquisas com seres humanos conforme o previsto na Resolução 466/12 do Conselho Nacional de Saúde, Ministério da Saúde.

O estudo obteve a autorização do Comitê de Ética em Pesquisa da Universidade Estadual do Sudoeste da Bahia conforme parecer n. 957.553.

\section{Resultados}

\section{Características sociodemográficas}

A média de idade dos pacientes foi de 50,5 anos $( \pm 14,9)$. Dentre os participantes, houve predomínio do sexo masculino (64,1\%), em união estável, vive com companheiro, (54,7\%) e católicos (59,4\%). Quanto à cor, $76,5 \%$ se autodeclararam como não brancos e $23,5 \%$ como brancos. A grande maioria vive em área urbana (96,5\%), acompanhados de familiares (84,7\%) e com renda familiar mensal de até 2 salários mínimos (83,5\%), sendo que entre estes, dois terços vivem com renda de até 1 salário. Frente à ocupação/regime de trabalho, todos se declararam inativos. Quando questionados sobre a escolaridade, $20 \%$ relataram nunca terem estudado (Tabela 1).

Tabela 1. Distribuição dos dados sociodemográficos dos pacientes renais crônicos hemodialíticos. Bahia, Brasil $2015(\mathrm{~N}=170)$.

\begin{tabular}{lcc}
\hline \hline Cexo & $\mathrm{N}$ & $\%$ \\
Masculino & 109 & 64,1 \\
Feminino & 61 & 35,9 \\
Situação conjugal & & \\
Solteiro & 56 & 32,9 \\
Casado+amasiado & 93 & 54,7 \\
Divorciado & 11 & 6,5 \\
Viúvo & 10 & 5,9 \\
Raça/Cor & & 23,5 \\
Branco & 40 & 76,5 \\
Não branco* & 130 & 59,4 \\
Religião & & 34,7 \\
Católica & 101 & 5,9 \\
Evangélica & 59 & 84,7 \\
Outras & 10 & \\
Com quem vive & & \\
Familiar & 144 & \\
\hline
\end{tabular}

6 


\begin{tabular}{|c|c|c|}
\hline Características Sociodemográficas & $\mathrm{N}$ & $\%$ \\
\hline Sozinho+ outros & 26 & 15,3 \\
\hline \multicolumn{3}{|l|}{ Área onde vive } \\
\hline Urbana & 164 & 96,5 \\
\hline Rural & 6 & 3,5 \\
\hline \multicolumn{3}{|l|}{ Renda familiar mensal } \\
\hline Até 1 salário & 98 & 57,6 \\
\hline Até 2 salários & 44 & 25,9 \\
\hline Acima de 2 salários & 28 & 16,5 \\
\hline \multicolumn{3}{|l|}{ Ocupação/Regime trabalho } \\
\hline Do lar & 3 & 1,8 \\
\hline Beneficiário sem $13^{\circ}$ & 91 & 53,5 \\
\hline Afastado temporariamente & 5 & 2,9 \\
\hline Aposentado invalidez ou contribuição & 71 & 41,8 \\
\hline \multicolumn{3}{|l|}{ Escolaridade } \\
\hline Não alfabetizado & 34 & 20,0 \\
\hline Alfabetizado** & 136 & 80,0 \\
\hline
\end{tabular}

Fonte: Elaborado pelos autores (2019).

*não branco: pardo, negro e amarelo. **alfabetizado: ensino fundamental, ensino médio e ensino superior.

\section{Características do estado de saúde e acesso aos serviços de saúde}

Na Tabela 2, podemos observar que $42,9 \%$ dos pacientes consideraram sua saúde atual como boa ou muito boa. Mais de $90 \%$ não possuíam plano de saúde e 32,4\% consideraram difícil a consulta médica. Em relação à data da última consulta, 57,1\% disseram ter sido nos últimos 3 meses. Referente ao tempo de tratamento hemodialítico, 48,2\% dos pacientes estavam em diálise por período de 1 a 5 anos. $O$ tempo médio de tratamento hemodialítico foi de 61,5 meses $( \pm 61,3)$, variando de 13 a 269 meses. Quando questionados sobre as duas comorbidades principais causadoras de doença renal crônica, 20\% negaram ser portadores de DM e/ou HAS, e a maioria (62,9\%) relatou ter HAS. Este último diagnóstico também foi relatado como causa isolada da perda da função renal na maioria $(57,8 \%)$ dos 121 pacientes que disseram conhecer a causa. Cabe ressaltar, que em $5 \%$ destes pacientes, o uso de medicamentos foi referido como o motivo da insuficiência renal crônica terminal. Hábitos de vida como tabagismo e/ou etilismo foram citados por 5,9\% dos pacientes. No que se refere a presença de limitação $17,1 \%$ dos pacientes afirmaram ter alguma forma de limitação para se locomover, sendo que em 75,9\% destes, foi físico-motora.

Tabela 2. Distribuição dos dados relacionados à saúde dos pacientes renais crônicos hemodialíticos, Bahia, Brasil, $2015(\mathrm{~N}=170)$.

\begin{tabular}{lcc}
\hline \multicolumn{1}{c}{ Variáveis } & $\mathrm{N}$ & $\%$ \\
\hline Possui plano de saúde & 12 & 7,1 \\
Sim & 158 & 92,9 \\
Não & & J Manag Prim Health Care, 2020;12:e27 \\
7 https://doi.org/10.14295/jmphc.v12.986 & &
\end{tabular}




\begin{tabular}{|c|c|c|}
\hline Variáveis & $\mathrm{N}$ & $\%$ \\
\hline \multicolumn{3}{|l|}{ Saúde atual } \\
\hline Muito boa/ boa & 73 & 42,9 \\
\hline Regular & 78 & 45,9 \\
\hline Muito ruim/ ruim & 19 & 11,2 \\
\hline \multicolumn{3}{|l|}{ Consultar com médico } \\
\hline Muito fácil/ fácil & 115 & 67,6 \\
\hline Muito difícil/ difícil & 55 & 32,4 \\
\hline \multicolumn{3}{|l|}{ Tempo em hemodiálise } \\
\hline 1 a 5 anos & 82 & 48,2 \\
\hline 5 a 10 anos & 49 & 28,8 \\
\hline Acima de 10 anos & 39 & 23 \\
\hline \multicolumn{3}{|l|}{ DM e/ou HAS } \\
\hline DM & 8 & 4,7 \\
\hline HAS & 107 & 62,9 \\
\hline Ambos & 21 & 12,4 \\
\hline Nenhum & 34 & 20 \\
\hline \multicolumn{3}{|l|}{ Afere pressão arterial fora da clínica } \\
\hline $\operatorname{Sim}$ & 115 & 67,6 \\
\hline Não & 55 & 32,4 \\
\hline \multicolumn{3}{|l|}{ Verifica glicemia fora da clínica } \\
\hline Sim & 28 & 16,5 \\
\hline Não & 142 & 83,5 \\
\hline \multicolumn{3}{|c|}{ Sabe a causa da perda funcional dos rins } \\
\hline Sim & 121 & 71,2 \\
\hline Não & 49 & 28,8 \\
\hline \multicolumn{3}{|l|}{ Causas da perda renal relatadas } \\
\hline HAS & 70 & 57,9 \\
\hline DM & 9 & 7,4 \\
\hline Hipertensão e Diabetes & 5 & 4,1 \\
\hline Medicamentosa & 6 & 5,0 \\
\hline Alcoolismo & 5 & 4,1 \\
\hline Outros & 26 & 21,5 \\
\hline \multicolumn{3}{|l|}{ Hábitos de vida } \\
\hline Tabagismo & 4 & 2,4 \\
\hline Etilismo & 5 & 2,9 \\
\hline Ambos & 1 & 0,6 \\
\hline Nenhum & 160 & 94,1 \\
\hline \multicolumn{3}{|l|}{ Última consulta } \\
\hline Menos de três meses & 97 & 57,1 \\
\hline Mais de três meses & 42 & 24,7 \\
\hline Não lembra & 31 & 18,2 \\
\hline
\end{tabular}

Fonte: Elaborado pelos autores (2019).

\section{Características relacionadas ao uso de medicamentos}

Foi contabilizado um total de 1.483 fármacos, com média 8,72 $( \pm 3,64)$, usados pelos pacientes, incluídos os prescritos e não prescritos. 0 número médio de medicamentos 
prescritos por paciente foi de $8,11( \pm 3,56)$, e o de não prescritos foi de $2,04( \pm 1,40)$, entre 51 pacientes que se automedicaram nos últimos trinta dias.

Para cerca de $70 \%$ dos participantes, o medicamento é reconhecido pelo nome (genérico ou comercial) e a maioria (80\%) faz uso da medicação sem ajuda de outras pessoas. A data de validade dos medicamentos e a consulta a bula costuma ser observada por aproximadamente $70 \%$ dos pacientes.

Aproximadamente $40 \%$ dos pacientes relataram ter usado medicamento sem prescrição médica em algum momento da vida (Tabela 3). Da mesma forma 35,3\% dos participantes afirmaram ter feito uso de algum chá ou planta com finalidade terapêutica e, dentro destes, os mais relatados foram erva-cidreira (Melissa officinalis), capim santo (Cymbopogon citratus) e erva-doce (Pimpinella anisum) com respectivamente, 27,4\%, 14,1\% e $9,4 \%$.

Tabela 3. Distribuição dos dados relacionados ao uso de medicamentos dos pacientes renais crônicos hemodialíticos, Bahia, Brasil 2015 ( $\mathrm{N}=170)$.

\begin{tabular}{|c|c|c|}
\hline Dados relacionados ao uso de medicamentos & $\mathrm{N}$ & $\%$ \\
\hline \multicolumn{3}{|l|}{ Como toma/usa seus medicamentos? } \\
\hline Sozinho & 136 & 80 \\
\hline Alguém ajuda & 34 & 20 \\
\hline \multicolumn{3}{|l|}{ Costuma ler a bula antes de usar medicamento? } \\
\hline $\operatorname{Sim}$ & 54 & 31,8 \\
\hline Não & 116 & 68,2 \\
\hline \multicolumn{3}{|l|}{ Costuma conferir a data de validade dos medicamentos? } \\
\hline Sim & 124 & 72,9 \\
\hline Não & 46 & 27,1 \\
\hline \multicolumn{3}{|l|}{$\begin{array}{l}\text { Já usou medicamento sem orientação ou receita médica } \\
\text { em algum momento da vida? }\end{array}$} \\
\hline Sim & 67 & 39,4 \\
\hline Não & 102 & 60 \\
\hline Não lembro & 1 & 0,6 \\
\hline
\end{tabular}

Fonte: Elaborado pelos autores (2019).

Os medicamentos usados, por prescrição e automedicação, foram classificados pela ATC, sendo que entre os prescritos, houve um predomínio do grupo terapêutico A com 387 medicamentos, seguidos, bem próximos, pelos grupos B e C, com respectivamente, 376 e 370 (Tabela 4). Não foi encontrado nenhum fármaco prescrito nos grupos terapêuticos L (antineoplásico e agentes imunomoduladores) e P (antiparasitários, inseticidas e repelentes). 
ISSN 2179-6750

Tabela 4. Distribuição dos medicamentos prescritos e não prescritos, segundo o subgrupo terapêutico, Bahia, Brasil, 2015.

\begin{tabular}{lcc}
\hline \multicolumn{1}{c}{ Prescritos por Subgrupo Terapêutico (ATC) } & $N$ & $\%$ \\
\hline A- Sistema digestivo e metabolismo & 387 & 28,1 \\
B- Sangue e órgãos hematopoiéticos & 376 & 27,3 \\
C- Sistema cardiovascular & 370 & 26,8 \\
D- Dermatológicos & 2 & 0,2 \\
G- Sistema genito-urinário e hormônios sexuais & 1 & 0,1 \\
H- Hormônios de uso sistêmico, exceto os sexuais e insulina & 6 & 0,4 \\
J- Antiinfecciosos de uso sistêmico & 8 & 0,6 \\
M- Sistema músculo-esquelético & 11 & 0,8 \\
N- Sistema nervoso & 62 & 4,5 \\
R- Sistema respiratório & 10 & 0,7 \\
S- Órgãos sensoriais & 3 & 0,2 \\
V- Vários & 142 & 10,3 \\
\hline Total & 1378 & 100 \\
\hline & $N$ & $\%$ \\
\hline A- Sistema digestivo e metabolismo & 30 & 29,1 \\
B- Sangue e órgãos hematopoiéticos & 7 & 6,8 \\
C- Sistema cardiovascular & 26 & 25,2 \\
H- Hormônios de uso sistêmico, exceto os sexuais e insulina & 1 & 1 \\
J- Anti-infecciosos de uso sistêmico & 1 & 1 \\
L- Agentes antineoplásicos e imunomoduladores & 1 & 1 \\
M- Sistema músculo-esquelético & 17 & 16,5 \\
N- Sistema nervoso & 10 & 9,7 \\
R- Sistema respiratório & 3 & 2,9 \\
V- Vários & 7 & 6,8 \\
\hline Total & 103 & 100 \\
\hline \hline
\end{tabular}

Fonte: Elaborado pelos autores (2019).

O medicamento mais frequente entre os prescritos foi a eritropoetina $(n=147)$, seguido por cloridrato de sevelamer $(n=139)$, hidróxido de ferro $(n=137)$ e calcitriol $(n=104)$, todos de uso na clínica de diálise. Dentre os 849 medicamentos prescritos e classificados como de uso domiciliar, os mais frequentes foram complexo B (8,2\%), vitamina C (8\%) e losartana (7,5\%). Os medicamentos classificados no grupo terapêutico $C$, da ATC, o de maior frequência foi a losartana $(n=64)$, seguido por clonidina $(n=48)$ e propranolol $(n=40)$. Dos fármacos do grupo terapêutico N, a dipirona foi a mais prescrita (27\%).

Entre os medicamentos utilizados por automedicação, prevaleceram os dos grupos A, C e M, respectivamente com 29,1\%, 25,2\% e 16,5\% (Tabela 4); sendo que no grupo A, o mais relatado foi o calcitriol (9,6\%), no grupo $C$, foi a clonidina $(6,7 \%)$ e no grupo $M$, a 
ISSN 2179-6750

nimesulida (5,7\%). Também esteve entre os mais citados, a dipirona, com 5,7\% de frequência entre todos os não prescritos. Um medicamento prescrito e um utilizado por automedicação não foram classificados na ATC em virtude de serem medicamentos considerados fitoterápicos ou de uso alternativo.

\section{Discussão}

Com o perfil farmacoterapêutico e clínico dos pacientes renais crônicos hemodialíticos foi possível caracterizar essa população regional o que pode servir de subsídio em ações estratégicas de saúde desde a atenção primária até a especializada. Neste estudo, constatou-se que a média de idade dos pacientes $(50,5 \pm 14,9$ anos) está em convergência com a predominante nos Censo 2013 e 2017 da SBN, 1,16 houve, também, um predomínio do sexo masculino, semelhante aos resultados encontrados em outros estudos. ${ }^{17-20}$ Percebe-se então, uma maior frequência de doentes dialíticos em uma idade economicamente produtiva, como também estes dados devem servir de alerta para melhorar o cuidado na atenção para com esta faixa etária da população. Já é conhecido que, em relação à população feminina, a masculina costuma retardar a procura por serviços de saúde, ${ }^{14}$ podendo ser esta uma das explicações para sua predominância em uma doença muitas vezes silenciosa, nos seus quadros iniciais.

O tratamento ideal para a DRC baseia-se em um diagnóstico precoce, implementação oportuna de tratamentos farmacológicos e não farmacológicos para população de risco (hipertensão arterial sistêmica e diabetes mellitus) bem como medidas para preservar a função renal, na assistência primária a saúde, antes do tratamento especializado. ${ }^{17,18,21}$ No Brasil, o tratamento medicamentoso é ofertado no mercado privado, assim como estão disponíveis gratuitamente na rede pública de serviços de saúde do SUS. Em um inquérito populacional sobre acesso a medicamentos para doenças crônicas não transmissíveis observou-se uma alta prevalência de acesso total, no entanto ocorrem diferenças significativas entre regiões, níveis socioeconômicos e condições de saúde, o que pode indicar a necessidade de promover melhorias da assistência farmacêutica, especialmente na região Nordeste, bem como da atenção à saúde aos mais pobres e mais doentes. ${ }^{22}$

Quanto à escolaridade, 20\% nunca estudaram e 57\% concluíram seu último ano de estudo no ensino fundamental, dados estes muito próximos aos de um estudo realizado em João Pessoa, Paraíba ${ }^{21}$ e semelhantes aos de outros, realizados em Taubaté, SP ${ }^{17}$ e Viseu, Portugal. $^{23}$ Neste mesmo estudo português, associado à baixa escolaridade, pequeno número de pacientes soube o nome das medicações que usou e mesmo assim, 30\% reduziram por conta própria, a quantidade que tomava. ${ }^{23}$ A DRC caracteriza-se por uma terapêutica complexa, exigindo mais das habilidades cognitivas dos pacientes, os quais, 
quanto mais inseridos dentro de um nível de escolaridade inferior e baixa compreensão do tratamento, piores resultados obtidos, ${ }^{24}$ inclusive com aumento da chance de abandono de tratamento. Estudo nacional sobre acesso a medicamentos mostrou que as maiores prevalências de baixa adesão estiveram associadas a adultos jovens; que nunca estudaram; residentes na região Nordeste e Centro-Oeste do País, sendo, portanto, importante ponto a ser considerado pelos gestores locais. ${ }^{25}$

Em relação à situação laboral e renda familiar, todos se encontravam inativos e 83\% recebiam até 2 salários mínimos. Em outros estudos, mais de $80 \%$ também se encontravam inativos ${ }^{23,24}$ e acima de $80 \%$ também possuíram renda familiar nesta faixa. ${ }^{21}$ Esta mesma faixa de renda foi encontrada em $23,4 \%$ dos doentes no estudo de Campos et al. ${ }^{26}$ Diante desta realidade, dentro do atual contexto econômico, o custo de um medicamento pode influenciar na adesão e, por consequência no resultado terapêutico, principalmente se for levado em consideração, que dentro de um núcleo familiar de baixa renda, muitas vezes a pessoa tem de optar por prioridades conforme a necessidade do momento, não aderindo ao tratamento farmacológico uma vez que o medicamento pode estar indisponível gratuitamente, já que o município sofre com episódios de desabastecimento na atenção básica, ou a farmácia é localizada em região geográfica de difícil acesso o que pode gerar impacto negativo na adesão ao tratamento. ${ }^{27}$

Em relação às características do seu estado de saúde e da assistência em saúde, 90\% tiveram como fonte pagadora de seu tratamento dialítico o SUS. No Censo 2017 da SBN, ${ }^{1}$ $82 \%$ dos pacientes também tiveram tratamento dialítico assistido com recursos do SUS. Para se entender melhor a DRC, principalmente na sua fase não dialítica, e sua associação com o envelhecimento e com doenças crônicas como DM e HAS, além de como estas são encaradas em seu tratamento, são encontrados dados de um estudo em que $60 \%$ dos pacientes só foram consultados por um nefrologista imediatamente ou apenas um mês antes de iniciarem a terapia dialítica e a maioria sequer sabia que DM e HAS poderiam evoluir com DRC, demonstrando a importância de ações de prevenção e tratamento oportuno na atenção primária a saúde. ${ }^{21} \mathrm{Em}$ um inquérito norte-americano, pacientes que possuíam seguro de saúde foram mais propensos a controlar esses fatores de risco modificáveis para DRC. ${ }^{28}$ Dos pacientes $45,9 \%$ considerou sua saúde atual como regular. Assim como no estudo de Oliveira Junior, ${ }^{21}$ houve predominância de pacientes que encontravam-se entre 1 e 5 anos em hemodiálise. Em um estudo sobre aderência e persistência à medicação prescrita, entre pacientes dialíticos, realizado por Park et al., ${ }^{6} \mathrm{o}$ tempo médio de diálise dos pacientes foi de 5,3 anos e em outro estudo português, foi de 4,89 anos com desvio padrão de 4,68 anos. Nakao, ${ }^{20}$ avaliando adesão em uma população

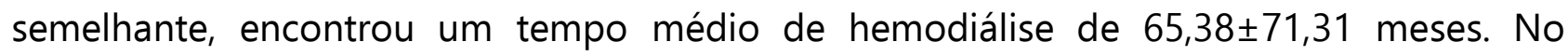


presente estudo, apesar de semelhante, o tempo médio de tratamento em diálise pode ter sido superestimado pela inclusão, apenas de pacientes com período mínimo de 1 ano de tratamento, o que exclui um grande número de pacientes que encerram o tratamento por óbito, geralmente nos 3 primeiros meses, devido a complicações clínicas concomitantes. ${ }^{18}$

Dos 170 participantes que responderam ao formulário, 121 atribuíram HAS como provável causa da perda do funcionamento de seus rins, representando $57,8 \%$, entre as mais frequentes citadas, acima dos 34\% encontrados no Censo de 2017 da SBN. Nefropatia diabética foi citada como causa isolada, não considerando, portanto, HAS associada, em apenas 7,4\% das causas, abaixo dos 31\% do Censo- 2017. ${ }^{1}$ Isto pode servir de alerta para os gestores locais, pela possibilidade destes pacientes diabéticos estarem vindo a falecer antes da fase dialítica, principalmente se considerarmos seu alto risco cardiovascular. $O$ tratamento oportuno e precoce da HAS e DM se coloca como importante ponto de intervenção para evitar ou postergar a evolução para DRC, promovendo uma melhora da qualidade de vida do paciente e permitindo melhor distribuição dos já limitados recursos do SUS, uma vez que o tratamento hemodialítico tem grande impacto econômico. ${ }^{8}$

Esta diferença encontrada em determinadas causas de doença renal crônica terminal (DRCt), pode ser explicada, primeiramente, pelo fato de terem sido autorrelatadas. Outras explicações que podem ser usadas na compreensão das causas de DRCt, inseridas no cenário atual do envelhecimento da população e no maior aparecimento de doenças crônicas são o déficit importante no acompanhamento clínico destas doenças, assim como a rara assistência do nefrologista, oferecida nas fases da DRC ainda não dialíticas. A baixa realização de biópsia renal é outro fator a se considerar, já que estes doentes costumam chegar ao nefrologista após o período recomendado para tal procedimento, com rins já atróficos. $^{21}$

Em relação ao uso de medicamentos, o número dos prescritos foi coerente em relação à média de outros estudos. ${ }^{16,21}$ Existem relatos diferentes, citando uma mediana de 10 a 12 medicamentos prescritos ou utilizados por paciente. ${ }^{4,29}$ Esta diferença pode variar de acordo com os critérios de seleção da amostra da população de cada estudo, além do fato de que, quando relatado sobre a utilização, muitas vezes não é discriminado, se inclui somente medicamento prescrito ou também os de automedicação, como neste estudo, em que a média destes foi de 2,04 $( \pm 1,40)$.

Apesar de 39,4\% terem relatado uso de automedicação em algum momento da vida, nota-se certa preocupação ou receio por parte dos pacientes perante a forma de utilização dos medicamentos, tendo em vista que a maior parte deles disse buscar informações na bula e verificar a data de validade dos medicamentos antes do uso e apenas um referiu ter aumentado a dose por conta própria. Cerca de 35\% já fizeram uso de medicamentos 
ISSN 2179-6750

alternativos como chás, com finalidade terapêutica. Doentes renais crônicos costumam usar frequentemente uma grande variedade de chás e sucos de frutas, por confiarem que estes são amplamente seguros e saudáveis, porém existem riscos principalmente relacionados a interação medicamentosa, sem falar que a comunicação entre os nefrologistas e os pacientes em relação a estes tipos de medicamentos de uso complementar é infrequente. ${ }^{30}$

Medicamentos de uso na clínica como eritropoetina, hidróxido de ferro e calcitriol, assim como cloridrato de sevelamer tiveram sua frequência de prescrição elevada, fato também evidenciado pelo Censo-2017 da SBN. ${ }^{1}$ Dentro dos medicamentos de uso cardiovascular, os três mais frequentemente prescritos foram losartana, clonidina e propranolol, todos com efeito anti-hipertensivo. Existe relato na literatura de que o uso de agentes do sistema renina-angiotensina, assim como beta-bloqueadores, foi associado com diminuição da mortalidade cardiovascular e por todas as causas, quando comparados os resultados com pacientes que não usaram estes medicamentos. ${ }^{31}$ Calcitriol e clonidina foram os dois medicamentos de maior frequência entre os utilizados por automedicação. Isto pode ser supostamente explicado, pelo fato do primeiro ter sido retirado da prescrição eletrônica, presente no prontuário eletrônico do paciente, na prescrição mensal mais recente, e o paciente possivelmente ainda não ter sido informado da suspensão, razão pela qual ter relatado o uso, principalmente na apresentação oral, já que na forma injetável, este prolongamento do uso não tinha como ocorrer, tendo em vista que, quando suspenso na prescrição eletrônica, não estaria incluído entre os que deveriam ser administrados pela equipe de enfermagem, na sala de diálise.

Quanto à clonidina, possivelmente foi prescrito em datas passadas e, se suspensa na prescrição eletrônica, tenha ocorrido erro na comunicação entre médico e paciente; outra probabilidade é que tenha sido prescrita em receituários manuscritos, não levados pelo mesmo por perda ou retenção na dispensação. Este prolongamento de uso de um medicamento previamente prescrito, mesmo que por falha na comunicação, se enquadra como automedicação. Uma comunicação clara e efetiva entre profissional da saúde e paciente é essencial para um cuidado seguro e de qualidade. Os problemas de comunicação estão entre os mais comumente encontrados em análise de causa raiz dos eventos sentinela, ${ }^{32}$ por isso os profissionais devem se preocupar em confirmar que o paciente compreendeu o tratamento proposto, mitigando os riscos relacionados a automedicação.

Neste estudo, observou-se uma baixa frequência $(0,67 \%)$ de uso de benzodiazepínicos e agonista do receptor ômega não benzodiazepínico (zolpidem), o que foi visto como boa prática, já que existe relato na literatura da associação do seu uso com maior mortalidade por todas as causas. ${ }^{33}$ 
ISSN 2179-6750

Pacientes renais crônicos dialíticos convivem frequentemente com dor, em decorrência de neuropatias periféricas, dores ósseas relacionadas ao hiperparatireoidismo avançado e artrites crônicas ${ }^{34}$, além de cefaleia. Isto pode explicar a frequência encontrada do uso de medicamentos com propriedades analgésicas (4,99\%), como dipirona e antiinflamatórios não esteroidais (AINEs) com uma representatividade de 2,1\% e 2,0\%, respectivamente, na população analisada.

Este estudo servirá de embasamento e informações para o planejamento de ações de prevenção direcionadas à DRC e à promoção da qualidade de vida. Iniciativas como essas são importantes para a Atenção Primária com vistas ao rastreamento de grupos de risco e fatores desencadeantes, além de contribuir para o planejamento da assistência farmacêutica, de forma a promover a adesão ao tratamento medicamentoso para o retardamento da progressão da doença, resultando em redução do sofrimento dos pacientes e dos custos financeiros do sistema de saúde.

\section{Limitações}

Nem todas as medicações prescritas aos pacientes na clínica são feitas exclusivamente por meio da prescrição eletrônica (apesar de ser uma prática dominante), não existindo protocolo específico.

Os resultados obtidos não podem ser generalizados para outras populações, pelo fato do estudo ter sido realizado em apenas um centro de nefrologia. Ademais, as respostas dos pacientes frente ao formulário foram espontâneas, não podendo ser confirmada a sua veracidade, como também podem ser influenciadas por viés de memória.

\section{Conclusão}

A caracterização de perfis farmacoterapêuticos e clínicos regionais é importante para oferecer subsídios para uma melhor assistência a todos os envolvidos com o tratamento dos pacientes renais crônicos hemodialíticos. Conscientes sobre as tendências de uso dos medicamentos, situações que necessitem de uma adequada intervenção, são mais facilmente e rapidamente reconhecidas.

Como pôde ser evidenciado, esses pacientes certamente se beneficiariam da atenção farmacêutica, principalmente se for considerada a presença da polifarmácia, da automedicação, como também o uso frequente de medicamentos de alto custo.

\section{Referências}

1. Thomé FS, Sesso RC, Lopes AA, Lugon JR, Martins CT. Brazilian chronic dialysis survey 2017. J Bras Nefrol. 2019;41(2):208-14. http://dx.doi.org/10.1590/2175-8239-JBN-2018-0178. 
2. Molina $M$, Allende LM, Ramos LE, Gutiérrez E, Pleguezuelo DE, Hernandéz ER, et al. CD19+ B-cells, a new biomarker of mortality in hemodialysis patients. Front Immunol. 2018;9:1221. http://dx.doi.org/10.3389/fimmu.2018.01221. eCollection 2018.

3. Chakraborty S, Ghosh S, Banerjea A, Raman R, Hazra A, Mandal SK. Prescribing patterns of medicines in chronic kidney disease patients on maintenance hemodialysis. Indian J of Pharmacol. 2016;48(5):586-90. http://dx.doi.org/10.4103/0253-7613.190760.

4. Menezes FG, Barreto DV, Abreu RM, Roveda F, Pecoits Filho RFS. Overview of hemodialysis treatment funded by the Brazilian Unified Health System-An economic perspective. J Bras Nefrol. 2015;37(3):367-78. http://dx.doi.org/10.5935/0101-2800.20150057.

5. Park H, Rascati KL, Lawson KA, Barner JC, Richards KM, Malone DC. Adherence and persistence to prescribed medication therapy among medicare part $D$ beneficiaries on dialysis: comparisons of benefit type and benefit phase. J Manag Care Spec Pharm. 2014;20(8):862-76. http://dx.doi.org/10.18553/jmcp.2014.20.8.862.

6. McIntyre C, McQuillan R, Bell C, Battistella M. Targeted deprescribing in an outpatient hemodialysis unit: a quality improvement study to decrease polypharmacy. Am J Kidney Dis. 2017;70(5):611-8. http://dx.doi.org/10.1053/j.ajkd.2017.02.374.

7. Nascimento RCRM, Álvares J, Guerra Junior AA, Gomes IC, Silveira MR, Costa EA, et al. Polifarmácia: uma realidade na atenção primária do Sistema Único de Saúde. Rev Saude Publica. 2017;51(Supl 2):19s. http://dx.doi.org/10.11606/s1518-8787.2017051007136.

8. Paula ECA, Mendes SJ. Farmacoeconomia e farmacoepidemiologia na gestão de recursos em saúde: primeiros resultados de uma revisão integrativa. J Manag Prim Health Care. 2019;11(Supl 1):e27s. https://doi.org/10.14295/jmphc.v11iSup.941.

9. Baldoni AO, Pereira LRL. The impact of Brazilian population aging to the health system under the pharmacoepidemiology vision: a narrative review. Rev Cienc Farm Basica Apl. 2011;32(3):313-21.

10. Avorn J. The promise of pharmacoepidemiology in helping clinicians assess drug risk. Circulation. 2013 Aug 13;128(7):745-8. http://dx.doi.org/10.1161/CIRCULATIONAHA.113.003419.

11. Estudo SABE: saúde, bem-estar e envelhecimento: condições de vida e saúde dos idosos do município de São Paulo [Internet]. c2006 [citado 21 fev. 2015]. Disponível em: http://www.fsp.usp.br/sabe/Extras/ Questionario_2006.pdf. 
12. Pesquisa nacional sobre acesso, utilização e promoção do uso racional de medicamentos no Brasil. Questionário do inquérito domiciliar adulto [Internet]. c2013 [citado 21 mar. 2015]. Disponível em: http://www.ufrgs.br/pnaum/documentos/questionarios1/PNAUM_Inq_Adulto.pdf/view.

13. Baldoni AO. Estudo de utilização de medicamentos em idosos atendidos pelo Sistema Único de Saúde (SUS) [dissertação]. [Ribeirão Preto]: Universidade de São Paulo; 2010. 127 f.

14. Martins GA, Acurcio FA, Franceschini SCC, Priore SE, Ribeiro AQ. Use of potentially inappropriate medications in the elderly in Viçosa, Minas Gerais State, Brazil: a population based survey. Cad Saude Publica. 2015;31(11):2401-12. http://dx.doi.org/10.1590/0102$311 \times 00128214$.

15. World Health Organization. Collaborating Centre for Drug Statistics Methodology, Guidelines for ATC classification and DDD assignment 2019. Oslo (Norway): WHO; 2018.

16. Sesso RC, Lopes AA, Thomé FS, Lugon JR, Santos DR. Inquérito brasileiro de diálise crónica 2013: análise das tendências entre 2011 e 2013. J Bras J Nefrol. 2014;36(4):476-81. http://dx.doi.org/10.5935/0101-2800.20140068.

17. Castro MCM, Aoki MVS, Domingos ES, Coutinho RCS, Silva CF, Couto JL et al. Determinantes da não-adesão medicamentosa nos pacientes em hemodiálise. J Bras Nefrol. 2009;31(2):89-95.

18. Melo FGC. Análise de sobrevida de pacientes renais crônicos em hemodiálise [dissertação]. [Fortaleza]: Universidade Federal do Ceará; 2006. $111 \mathrm{f}$.

19. Santos KK, Lucas TC, Glória JCR, Pereira Júnior AC, Ribeiro GC, Lara MOO. Epidemiological profile of chronic renal patients in treatment. J Nursing UFPE. 2018;12(9):2293-300. http://dx.doi.org/10.5205/1981-8963-v12i9a234508p2293-2300-2018.

20. Nakao, RT. Variáveis sociodemográficas, clínicas e psicológicas associadas à adesão à hemodiálise [dissertação]. [Ribeirão Preto]: Universidade de São Paulo; 2013. 112 p.

21. Oliveira Junior HM, Formiga FFC, Alexandre CS. Perfil clínico-epidemiológico dos pacientes em programa crônico de hemodiálise em João Pessoa - PB. J Bras Nefrol. 2014;36(3):36774. https://doi.org/10.5935/0101-2800.20140052.

22. Tavares NUL, Bertoldi AD, Mengue SS, Arrais PSD, Luiza VL, Oliveira MA, et al. Fatores associados à baixa adesão ao tratamento farmacológico de doenças crônicas no Brasil. Rev Saude Publica. 2016;50(supl 2):10s. https://doi.org/10.1590/S1518-8787.2016050006150. 
23. Sousa, MEP. Adesão ao tratamento medicamentoso da pessoa portadora de insuficiência renal crónica em hemodiálise [dissertação]. [Viseu]: Escola Superior de Saúde de Viseu; 2012. $110 \mathrm{f}$.

24. Martins MV, Mata AMLL, Camerini DM, Murta AR. Fatores que influenciam a adesão ao tratamento de hemodiálise para doença renal crônica. Rev Cient Faminas. 2017;12(1):5-15.

25. Oliveira MA, Luiza VL, Tavares NUL, Mengue SS, Arrais PSD, Farias MR, et al. Acesso a medicamentos para doenças crônicas no Brasil: uma abordagem multidimensional. Rev Saude Publica. 2016;50(supl 2):6s. http://dx.doi.org/10.1590/s1518-8787.2016050006161.

26. Campos CS, Santos KB, Ferreira GF, Bastos KV. Perfil epidemiológico dos pacientes em lista única de espera para transplante renal na Cidade de Juiz de Fora. HU Rev. 2017;43(4):40713.

27. Santos JB, Almeida PHRF, Lemos LB, Lemos GS. Evidências clínicas da adesão medicamentosa de pacientes dialíticos crônicos. J Nurs UFPE. 12(12):3254-62. http://dx.doi.org/10.5205/1981-8963-v12i12a236074p3254-3262-2018

28. Hall YN, Rodriguez RA, Boyko EJ, Chertow GM, O'Hare AM. Characteristics of uninsured Americans with chronic kidney disease. J Gen Intern Med. 2009;24(8):917-22. http://dx.doi.org/10.1007/s11606-009-1028-3.

29. Ribeiro PRS, Batista TS. Adesão ao tratamento farmacológico anti-hipertensivo de pacientes em hemodiálise. Rev Cienc Farm Basica Apl. 2015;36(2):201-12.

30. Chiu YW, Teitelbaum I, Misra M, Leon EM, Adzize T, Mehrotra R. Pill burden, adherence, hyperphosphatemia, and quality of life in maintenance dialysis patients. Clin J Am Soc Nephrol. 2009;4(6):1089-96. http://dx.doi.org/10.2215/CJN.00290109.

31. Iseki K, Shoji T, Nakai S, Watanabe $Y$, Akiba T, Tsubakihara Y. Higher survival rates of chronic hemodialysis patients on anti-hypertensive drugs. Nephron Clin Pract. 2009;113(3):c183-90. http://dx.doi.org/10.1159/000232600.

32. Institute of Medicine [IOM]. The healthcare imperative: lowering costs and improving outcomes: workshop series summary. Washington, DC: The National Academies Press; 2010 [citado 32 nov. 2019]. Disponível em: http://books.nap.edu/catalog.php?record_id=12750.

33. Winkelmayer WC, Mehta J, Wang PS. Benzodiazepine use and mortality of incident dialysis patients in the United States. Kidney Int. 2007;72(11):1388-93. http://dx.doi.org/10.1038/sj.ki.5002548. 
34. Andreucci VE, Fissell RB, Bragg-Gresham JL, Ethier J, Greenwood R, Pauly M, et al. Dialysis Outcomes and practice patterns study (DOPPS) data on medications in hemodialysis patients. Am J Kidney Dis. 2004;44(Suppl 2):61-7. https://doi.org/10.1053/j.ajkd.2004.08.013.

\section{Minicurrículo}

Lucas Brasileiro Lemos | ORCiD: 0000-0001-8355-0101

Médico pela Universidade do Vale do Sapucaí. Especialista em Nefrologia pela Universidade de Campinas - UNICAMP. Mestre em Ciências da Saúde pela Universidade Estadual do Sudoeste da Bahia - UESB, Brasil.

Gabriela Silva Moraes | ORCiD: 0000-0002-9487-1127

Farmacêutica pela Universidade Estadual do Sudoeste da Bahia - UESB, Brasil.

Gisele da Silveira Lemos | ORCiD: 0000-0001-8987-0245

Farmacêutica pela Universidade Federal de Minas Gerais - UFMG. Especialista em Assistência Farmacêutica pela Universidade Federal de Santa Catarina e em Segurança do Paciente pela Escola Nacional de Saúde Pública Sergio Arouca. Doutora em Medicamentos e Assistência Farmacêutica pela Universidade Federal de Minas Gerais - UFMG, Brasil.

Adriana Alves Nery | ORCiD: :0000-0002-1093-1437

Enfermeira pela Universidade Estadual do Sudoeste da Bahia - UESB. Mestre em Enfermagem pela Universidade Federal do Estado do Rio de Janeiro. Doutora em Enfermagem pela Escola de Enfermagem de Ribeirão Preto da Universidade de São Paulo. Professora do Departamento de Saúde II, docente e coordenadora do Programa de Pós-graduação em Enfermagem e Saúde da UESB. 